\title{
Prolonged High-Fat Diet Feeding of WHHLMI Rabbits does Not Aggravate Metabolic or Cardiac Dysfunction
}

\author{
Bianca Hemmeryckx ${ }^{1}$, Yuanbo Feng ${ }^{2}$, Liesbeth Frederix ${ }^{1}$, Marleen Lox ${ }^{1}$, Jun Wu ${ }^{3}$, Ward Heggermont ${ }^{1,4}$, \\ Yicheng $\mathrm{Ni}^{2}$, H. Roger Lijnen ${ }^{*}$
}

${ }^{1}$ Center for Molecular and Vascular Biology, Department of Cardiovascular Sciences, China

${ }^{2}$ Radiology, Department of Imaging and Pathology, KU Leuven, Leuven, Belgium

${ }^{3}$ Ultrasound Diagnostic Department, the second affiliated hospital of Dalian Medical University, Dalian, China

${ }^{4}$ Cardiovascular Research Centre Aalst, OLV Hospital, Aalst, Belgium

\begin{abstract}
:
Myocardial infarction (MI)-prone Watanabe hereditary hyperlipidemic (WHHLMI) rabbits were reported to spontaneously but slowly develop MI, associated with enhanced coronary atherosclerosis and metabolic syndrome features. Our aim was to accelerate these processes by exposing the rabbits to a high-fat diet (HFD) that was previously shown to induce insulin resistance and cardiac injury in rabbits.

HFD feeding for one year was indeed associated with development of obesity ( $37 \%$ increases in body weight, as compared to $3.7 \%$ for control standard-fat diet, SFD). On HFD, liver weight and hepatic triglyceride content were significantly higher as compared to SFD, with mild steatosis. Glucose and insulin levels were not markedly different on SFD or HFD, however after 1 year of diet treatment, HFD-fed animals were more glucose intolerant as compared to SFD-fed rabbits. On SFD, the rabbits developed marked thoracic aorta stenosis and calcification of atherosclerotic lesions, which were not aggravated by HFD feeding. Cardiac function analysis by MRI and transthoracic echocardiography did not reveal significant differences between SFD- and HFD- fed rabbits.

Thus, the metabolic and cardiac phenotype of WHHLMI rabbits was not aggravated by HFD feeding for one year, and the rabbits did not spontaneously develop diabetes or myocardial infarction.
\end{abstract}

Keywords: WHHLMI rabbits; Obesity; Hepatosteatosis; Atherosclerosis; Metabolism

\section{Introduction}

Obesity has become a worldwide epidemic: according to the World Health Organization (WHO) in 2016 more than 1.9 billion adults were overweight, while over 650 million were obese (http://www.who.int/mediacentre/factsheets/fs311/en/). Obesity, an unbalance between energy consumption and energy expenditure, leads to excessive adipose tissue (AT) accumulation and predisposes to the development of obesity-related comorbidities such as insulin resistance, type-2 diabetes, non-alcoholic fatty liver disease, and cardiovascular problems including atherosclerosis. Atherosclerosis is a complex disease leading to the buildup of fatty streaks inside the arteries; it frequently starts early in life, progresses relatively slow and predisposes to the development of cardiovascular diseases (CVD).

Several murine (reviewed by ${ }^{[1]}$ ) and rabbit models (reviewed by ${ }^{[2]}$ ) are being used to investigate the pathogenic mechanisms leading to atherosclerotic plaque development and to identify potential new therapeutic interventions. Myocardial infarction-prone Watanabe heritable hyperlipidemic (WHHLMI) rabbits were reported to spontaneously develop severe coronary atherosclerosis and MI due to selective breeding from the WHHL rabbits, a strain that shows hypercholesterolemia, resulting in coronary atherosclerosis due to a deficiency in the low-density lipoprotein (LDL) receptors ${ }^{[3]}$. Shiomi et al. ${ }^{[4]}$ also reported that these WHHLMI rabbits are a good model for the metabolic syndrome. 17- to 21-months old rabbits showed higher accumulation of RAM-11 (macrophages/monocytes)-positive mesenteric (MES) AT and lower plasma insulin levels as compared to adult WHHLMI rabbits ${ }^{[4]} .16$ weeks exposure of WHHL rabbits to a high-fat and high-fructose diet (HFFD) induced an elevation of plasma lipid levels, impaired glucose tolerance, accumulation of AT and accelerated coronary atherosclerosis in these animals ${ }^{[5]}$.
Received date: March 17, 2018 Accepted date: May 17, 2018 Published date: May 23, 2018

*Corresponding author: Prof. Dr. H. Roger Lijnen, Center for Molecular and Vascular Biology, KU Leuven, Campus Gasthuisberg, CDG, $9^{\text {th }}$ floor, Herestraat 49, Box 911, 3000 Leuven, Belgium, Tel 32-16-372053; E-mail: roger.lijnen@kuleuven.be

Citation: Lijnen, R., et al. Prolonged High-Fat Diet Feeding of WHHLMI Rabbits does Not Aggravate Metabolic or Cardiac Dysfunction. (2018) J diab Obes 5(1): 10- 17.

Copyright: (C) 2018 Lijnen, R. This is an Open access article distributed under the terms of Creative Commons Attribution 4.0 International License. 
Our aim was to characterize the long-term effects of high-fat diet (HFD)-induced obesity on atherogenesis, MI development and on metabolic syndrome features by keeping WHHLMI rabbits for an extended period of time (12 months) on this diet, that has been shown to slowly decrease insulin resistance ${ }^{[6]}$. The effect of this diet as compared to a control diet was investigated on metabolism and on the hepatic and cardiovascular system.

\section{Materials and Methods}

\section{Animal model}

Male WHHLMI rabbits at the age of 22 weeks were obtained from Dr. Shiomi (Institute for Experimental Animals, Kobe University School of Medicine, Japan). Upon arrival at our animal facility they were allowed to acclimatize for 3 weeks. Animals were kept single-housed in stainless steel cages in a temperature, humidity and light-controlled (12hrs night ( $7 \mathrm{pm})$ /day (7 am) cycle) environment and they were provided with $120 \mathrm{~g}$ of food (K-H, Ssniff, Soest, Germany) daily and had ad libitum access to drinking water. The rabbits received once a week a small bundle of hay and had a wooden block as enrichment. Animals were randomized into two groups with matching body weights (age 27 weeks): a control group of WHHLMI rabbits received $80 \mathrm{~g}$ of a standard fat diet (SFD; $9.4 \% \mathrm{kcal}$ as fat; caloric value $0.27 \mathrm{kcal} / \mathrm{g}$; customized by Ssniff, according to $\left.{ }^{[7]}\right)$, while the other group of rabbits was exposed to $120 \mathrm{~g}$ of the HFD (SFD supplemented with $6.7 \%$ corn oil and $3.3 \%$ lard; $32.5 \% \mathrm{kcal}$ as fat; caloric value $1.1 \mathrm{kcal} / \mathrm{g}$; customized by Ssniff). Body weight was monitored every week and food intake daily for one year. Blood was collected from the ear vein on $3.8 \%$ citrate after an overnight fast at the start and after 12 months exposure to the diets. A glucose tolerance test was performed after 41 weeks and after 12 months of HFD/SFD loading. To evaluate cardiac function, magnetic resonance imaging (MRI) and transthoracic echocardiography were performed at baseline and at the end of the study. Animals were euthanized with an intravenous (iv) injection of 2-4 $\mathrm{ml}$ undiluted sodium pentobarbital (Nembutal, Abbott Laboratories, North Chicago, IL, USA). Organs including subcutaneous inguinal (SC) and abdominal gonadal (GON) fat, liver, kidney, heart, and thoracic aorta were excised, weighed and processed for gene expression analysis (liver, heart), and/or histology (liver, heart and thoracic aorta).

All animal procedures were approved by the Ethical Committee of the KU Leuven (P076/2013), and performed in accordance with the guidelines from Directive 2010/63/EU of the European Parliament on the protection of animals used for scientific purpose and conform the guidelines of the Declaration of Helsinki.

\section{MRI \\ Scanning}

Using a 16-channel phased array knee coil, cMRI was performed on the anesthetized rabbits using a 3.0T clinical Siemens MRI scanner (Trio, Siemens, Erlangen, Germany) with a maximum gradient capability of $45 \mathrm{mT} / \mathrm{m}$ triggered by ECG and gated by respiration using a small animal monitoring and gating system (SA Instruments, Inc. Stony Brook, NY, USA). The two ECG electrodes were attached to the shaved thorax skin and to the left leg. The respiration sensor was attached to the mid-section of the abdomen of the rabbit, which was placed supinely in a holder and gas-anesthetized with $2 \%$ isoflurane in the mixture of $20 \%$ oxygen and $80 \%$ room air, through a mask connected via a tube to a ventilation instrument (Harvard Apparatus, Holliston, MA, USA). All images were acquired during free breathing of the animal. Eight short-axial slices of the left ventricle (LV) were collected with a slice thickness of $3.0 \mathrm{~mm}$ without gap for cMRI sequences. Turbo spin echo sequence of black blood imaging was applied for cardiac morphology with parameters TR: 621 750 ms, TE: 15 74 ms, FOV: $240 \times 195 \mathrm{~mm}^{2}$, FA: 180 , and in-plane resolution: $0.9 \times 0.9 \mathrm{~mm}^{2}$. The cine-MR images were acquired on True fast imaging with steady state precession in the short-axis, vertical long-axis and horizontal long-axis planes for displaying cardiac contraction. Each cine-MRI consisted of 25 frames, spaced equally across the cardiac cycle, with the acquisition time of $2.5 \mathrm{~min}$, the scan parameters TR: $357 \mathrm{~ms}$, TE: 1.6 ms, FOV: $240 \times 195 \mathrm{~mm}^{2}$, FA: $60^{\circ}$, spatial resolution: $1.2 \times 0.9$ $\mathrm{mm}^{2}$. The contrast delayed-enhancement images (CE) were acquired by a 3D segmented k-space inversion recovery turbo fast low angle shot sequence 20 minutes after an iv bolus injection of megluminegadoterate [(Gd-DOTA) Dotarem, Guerbet, France] at $0.2 \mathrm{mmol} / \mathrm{kg}$ with parameters TR: $396 \mathrm{~ms}$, TE: $1.54 \mathrm{~ms}$, TI: $360 \mathrm{~ms}, \mathrm{FOV}: 240 \times 180 \mathrm{~mm}^{2}$, FA: $15^{\circ}$, and in-plane resolution: $1.1 \times 0.8 \mathrm{~mm}^{2}$.

\section{Image analysis}

cMRI images were read using an off-line workstation with dedicated software (SyngoMR A30, Siemens). The assessment and quantification of MI size and LV function in CE and Cine-MRI images were done using the software SEGMENT (Medviso AB, Lund, Sweden). The endocardial and epicardial borders were manually traced in the end-diastolic and end-systolic short-axis Cine images. Papillary muscles were included in the myocardium. LV end-diastolic volume (EDV), end-systolic volume (ESV), stroke volume (SV), ejection fraction (EF), cardiac output $(\mathrm{CO})$ and mass were measured according to standard methods $^{[8]}$. Regional LV function was assessed by measuring wall thickening from end-diastolic phase to end-systolic phase in six clockwise sectors on the mid-ventricle section of Cine images.

\section{Transthoracic echocardiography}

Transthoracic echocardiographic examinations were performed on ketamin (injected intramuscularly; $15 \mathrm{mg} / \mathrm{kg}$; Nimatek; Eurovet Animal Health B.V., Bladel, The Netherlands) and xylazin (injected intramuscularly; $2.5 \mathrm{mg} / \mathrm{kg}$; Xyl-M; V.M.D. n.V., Arendonk, Belgium) anesthetized rabbits at baseline, and after 12 month exposure to the diets (SFD/HFD), using a 10S transducer (4.4 - $10 \mathrm{Mhz}$ ) (GE Healthcare, Machelen, Belgium) on a Vivid 7 ultrasound machine (GE Healthcare). Rabbits were scanned either in a recumbent position with the shaved right area over the cutout in a wooden exam plank on top of a surgical table or in an upright chest position. LV diameter at end-diastole (LVIDd) and end-systole (LVIDs), muscle thickness in diastole (IVSd) and in systole (IVSs) and LV posterior wall thickness in end-diastole (LVPWd) and end-systole (LVPWs) were measured at three levels: at the level of the mitral valve (mv), papillary muscle (pm) and apex (ap). In addition, the long axis diameter in end-diastole (LAXd) and systole (LAXs) were obtained as well. End-diastol- 
ic volume $\left(\mathrm{EDV}\right.$; formula EDV $=\left(\left(\right.\right.$ LVIDd_mv ${ }^{2}+$ LVIDd_pm ${ }^{2}+$ LVIDd_ap $\left.\left.{ }^{2}\right)^{*} \mathrm{LAXd}^{*} \Pi / 18\right)$ and end-systolic volume (ESV; formula $\overline{E S V}=\left(\left(\text { LVIDs_mv }{ }^{2}+\text { LVIDs } \text { pm }^{2}+\text { LVIDs_ap }\right)^{*}\right.$ LAX$\left.\mathrm{s}^{*} \Pi / 18\right)$, ejection fraction $(\mathrm{EF}$; formula $\mathrm{EF}=((\mathrm{EDV}-\mathrm{ESV}) /$ EDV)*100), stroke volume (SV; formula SV $=$ EDV - ESV) and cardiac output $(\mathrm{CO}$; formula $\mathrm{CO}=$ heart rate $(\mathrm{HR}) * \mathrm{SV})$ were calculated $^{[9]}$. The measurements for all parameters at the end of the diet exposure are reported as a percentage of the baseline measurement for each rabbit. Subsequently, an average per group was calculated for each parameter.

\section{Analyses \\ Blood glucose concentrations were measured using Glucocard strips (28350, Menarini Diagnostics, Firenze, Italy) and tri- glyceride, total cholesterol, HDL-cholesterol and cardiac Tro- ponin $\mathrm{T}$ levels were evaluated using routine clinical assays (en- zymatic methods). The LDL-cholesterol levels were calculated as total cholesterol - HDL-cholesterol - (triglycerides/5). The content of triglycerides in the liver was quantified with the tri- glycerides FS* kit (Diasys, Holzheim, Germany), after prepara- tion of liver tissue as described ${ }^{[10]}$.}

\section{Glucose tolerance test}

After an overnight fast, glucose $(0.6 \mathrm{~g} / \mathrm{kg}$ body weight) was injected into the catheterized ear vein. Blood was collected via the catheterized artery (of the other ear) for glucose measurement before and at 5-120 minutes after injection. Data are represented as areas under the curves (AUC) of glucose level versus time.

\section{Gene expression analysis}

Taqman gene expression assays (Thermo Fischer Scientific BVBA, Erembodegem, Belgium) were used to analyze mRNA levels of the following genes in liver or heart tissue by quantitative RT-PCR: cluster of differentiation 36 (CD36, OC03395924_ $\mathrm{m} 1$ ), fatty acid synthase (FAS, OC04096820_m1), tissue inhibitor of matrix metalloproteinases-1 (TIMP-1, OC03397606_m1), transforming growth factor b1 (TGF- $\beta 1$, OC04176122_u1), collagen type 1 $\alpha-1$ (COL1a1, OC03396073_g1), arginase 1 (Arg1, OC03397217_m1), tumour necrosis factor- $\alpha$ (TNF- $\alpha$, OC03397715_m1), interleukin-6 (IL-6, OC04097053_m1), monocyte chemoattractant protein-1 (MCP-1, OC03823583 s1), smooth muscle $\alpha$-actin (SMA, OC03399261_m1) and $\beta$-actin (OC03824857_g1) according to a protocol described elsewhere $^{[11]}$. For each animal, samples were applied in duplicate and the results were averaged. Data were obtained as cycle threshold $(\mathrm{Ct})$ values and were normalized to the housekeeping gene $\beta$-ac$\operatorname{tin}\left(\Delta \mathrm{Ct}=\mathrm{Ct}_{\text {target }}-\mathrm{Ct}_{\beta \text {-actin }}\right)$ for liver tissues and to the housekeeping gene glyceraldehyde 3-phosphate dehydrogenase (GAPDH) $\left(\left(\Delta \mathrm{Ct}=\mathrm{Ct}_{\text {target }}-\mathrm{Ct}_{\mathrm{GAPDH}}\right)\right.$ for heart tissues. For each group the data are represented as means of the $\Delta \mathrm{Ct}$ values $\pm \mathrm{SEM}$.

\section{Histology}

The thoracic aorta $(2-3 \mathrm{~cm}$ portion from the diaphragm was used for histology and rest of the segment was used to stain atherosclerotic plaques using the dye Oil Red O) was cut into 5 $\mathrm{mm}$ rings of which the second and every other ring was used to prepare cross-sectional sections. Liver $(8 \mu \mathrm{m})$, heart $(5 \mu \mathrm{m})$ and thoracic aorta $(8 \mu \mathrm{m})$ sections, cut on a HM360 microtome (Microm, Walldorf, Germany), were deparaffinized in xylene, followed by series of decreasing ethanol concentrations and distilled water. To study general morphology and calcification of the atherosclerotic plaques, H\&E and Von Kossa staining were done on thoracic aorta sections. Heart sections were stained with Sirius Red to study fibrosis. Macrophage content of the atherosclerotic plaques was determined by incubating sections with the primary mouse anti-rabbit RAM-11 monoclonal antibody (1/50; M0633, DAKO, Leuven, Belgium) overnight at room temperature in Tris-NaCl-blocking buffer (TNB) $(0.1 \mathrm{M}$ Tris- $\mathrm{HCl} \mathrm{pH} 7.5,0.15 \mathrm{M} \mathrm{NaCl}, 0.5 \%$ blocking reagent (TSA BT kit, NEL700001, Perkin Elmer, Boston, MA)). Prior to this incubation, macrophage antigens were released by immersion in Target Retrieval solution (S1699, DAKO) for $20 \mathrm{~min}$ at $95^{\circ} \mathrm{C}$. Subsequently, endogenous peroxidase activity was blocked by a 20 min incubation in methanol containing $0.3 \% \mathrm{H}_{2} \mathrm{O}_{2}$ (Merck, Overijse, Belgium) at RT. Aspecific binding to the biotin-labeled secondary goat anti-mouse antibody (1/300, E0433, DAKO) was blocked by incubating sections with $20 \%$ pre-immune goat serum (X0907, DAKO) for $45 \mathrm{~min}$ in TNB. The biotin label on the aorta and aortic arch sections was detected by avidin-biotin-peroxidase complex (Vector Laboratories Ltd, Peterborough, United Kingdom). The peroxidase signal was then visualized with 3, 3'-diaminobenzidine (DAB) (Sigma-Aldrich) at RT and subsequently counterstained with Harris hematoxylin (VWR, Leuven, Belgium). Sections were then run through a series of increasing percentages of ethanol and finally xylene. The sections were mounted with DEPEX (VWR, Leuven). Negative control included a section incubated with buffer alone.

\section{Microscopic analysis}

Images of H\&E-, Von Kossa and RAM-11 antibody-stained thoracic sections were obtained with an Axiovert 200M Imaging microscope (Zeiss, Jena, Germany) with normal light and the Axiovision Rel. 4.8. software (Zeiss) at x200 magnification. The degree of arterial stenosis (\% of plaque filling the arterial lumen) in these sections was determined by analyzing images of H\&E-stained sections ( $n=9-14$ /animal). Using the National Institutes of Health Image $\mathrm{J}$ software, the total area of the lumen (plaque area and area of unfilled lumen) and the area of the empty lumen was delineated. The degree of stenosis was then calculated as total area of lumen - area of empty lumen divided by the total area of lumen multiplied by 100 . The degree of calcification of the plaque was investigated by analyzing images obtained from Von Kossa stained sections. Macrophage accumulation in the plaques was determined by analyzing images from sections stained with the mouse anti-rabbit RAM-11 monoclonal antibody. Both DAB-colored images (Von Kossa ( $\mathrm{n}=18$-39/animal); RAM-11 ( $\mathrm{n}=19-48 /$ animal)) were analyzed by segmenting the brown color of DAB in the total plaque area. The degree of calcification and macrophage accumulation were then shown as the percentage of stained area to the total area. The presence of fibrosis in Sirius Red stained cardiac sections was analyzed by segmenting the red color of collagen in each cardiac section. The degree of fibrosis was then calculated as the percentage of stained area to the total area. 


\section{Statistical analysis}

Data are shown as means \pm SEM for the number of animals studied. Differences between all groups were analyzed using the nonparametric Mann-Whitney $U$ test. Values of $p<0.05$ were considered statistically significant. The statistical analyses were performed with GraphPad Prism 6 software (GraphPad, La Jolla, CA USA).

\section{Results}

\section{Body composition and metabolism}

Exposure of 7 months old WHHLMI rabbits $(n=9)$ to a HFD for 1 year did not affect their survival rate (69\% survival at 595 days versus $71 \%$ on $\operatorname{SFD}(\mathrm{n}=9) ; \mathrm{P}=0.62)$. These rabbits gained about $37 \%$ of their initial body weight, in contrast to $3.7 \%$ for the SFD-fed rabbits (Table 1). SC and GON fat mass as well as liver and kidney weight were significantly higher after HFD feeding, whereas heart weight was not affected. Food intake was considerably higher in the rabbits fed with HFD versus SFD (Table 1).

Table 1: Effect of a high-fat and high-cholesterol diet (HFD) on the body composition of coronary atherosclerosis- and myocardial infarction-prone Watanabe heritable hyperlipidemic (WHHLMI) rabbits

\begin{tabular}{|c|c|c|}
\hline & SFD $(n=8-9)$ & $\operatorname{HFD}(n=6-9)$ \\
\hline \multicolumn{3}{|l|}{ Body weight (kg) } \\
\hline start & $2.7 \pm 0.049$ & $2.7 \pm 0.034$ \\
\hline end & $2.8 \pm 0.063$ & $3.7 \pm 0.15^{\mathrm{aa}}$ \\
\hline Body weight gain $(\mathrm{kg})$ & $0.056 \pm 0.049$ & $0.96 \pm 0.14^{\text {aа }}$ \\
\hline Calorie intake (kcal/day) & $229 \pm 0.18$ & $377 \pm 4.5^{\text {aa }}$ \\
\hline SC fat $(\mathrm{g})$ & $15 \pm 2.4$ & $57 \pm 6.3^{\text {aa }}$ \\
\hline GON fat $(\mathrm{g})$ & $2.9 \pm 0.47$ & $18 \pm 2.5^{\text {aa }}$ \\
\hline Liver $(\mathrm{g})$ & $65 \pm 2.1$ & $93 \pm 7.9^{\text {aa }}$ \\
\hline Kidneys (g) & $17 \pm 0.67$ & $21 \pm 1.3^{\mathrm{a}}$ \\
\hline Heart (g) & $8.3 \pm 0.57$ & $8.8 \pm 0.22$ \\
\hline
\end{tabular}

Data are means \pm SEM for $\mathrm{n}$ animals. ${ }^{\mathrm{a}} \mathrm{P}<0.05$ and ${ }^{\mathrm{a}} \mathrm{P}<0.001$ versus SFD (Mann-Whitney U test). Abbreviations: (SC) subcutaneous, (GON) gonadal.

Blood glucose and plasma insulin levels did not markedly change upon SFD or HFD feeding, and were comparable for both diets (Table 2). Glucose levels at 11 weeks, and at 3 and 6 months post HFD feeding were statistically significantly lower as compared to SFD-fed rabbits (11 weeks: $96 \pm 1.4 \mathrm{mg}$ / dl versus $104 \pm 2.0 \mathrm{mg} / \mathrm{dl}, \mathrm{P}=0.0073 ; 3$ months: $96 \pm 1.3 \mathrm{mg}$ / dl versus $103 \pm 1.7 \mathrm{mg} / \mathrm{dl}, \mathrm{P}=0.0091 ; 6$ months: $99 \pm 1.4 \mathrm{mg} / \mathrm{dl}$ versus $109 \pm 3.0 \mathrm{mg} / \mathrm{dl}, \mathrm{P}=0.013)$. Glucose tolerance tests after 41 weeks of diet revealed no change in glucose tolerance between the two groups (AUC HFD: 24,637 \pm 907 versus AUC for SFD: $23,921 \pm 1,477 ; \mathrm{P}=0.95)$. However, after one year glucose intolerance was observed in the HFD group, as confirmed by a 1.3-fold higher AUC for the HFD (27,026 $\pm 1,778$ versus 21,461 $\pm 590 ; \mathrm{P}=0.030)$.

At the end of the experiments, plasma levels of total cholesterol, LDL-cholesterol or triglycerides were comparable for both diets, whereas HDL-cholesterol was enhanced on the HFD (Table 2).
Table 2: Effect of a high-fat and high-cholesterol diet (HFD) on metabolic and liver function parameters of coronary atherosclerosis- and myocardial infarction-prone Watanabe heritable hyperlipidemic (WHHLMI) rabbits

\begin{tabular}{|c|c|c|}
\hline & $\operatorname{SFD}(n=7-8)$ & $\operatorname{HFD}(\mathrm{n}=3-8)$ \\
\hline \multicolumn{3}{|l|}{ Glucose (mg/dL) } \\
\hline start & $108 \pm 3.4$ & $106 \pm 3.2$ \\
\hline end & $115 \pm 4.8$ & $108 \pm 14$ \\
\hline \multicolumn{3}{|l|}{ Insulin (mU/L) } \\
\hline start & $12 \pm 2.1$ & $9.0 \pm 1.6$ \\
\hline end & $6.2 \pm 1.3$ & $10 \pm 5.3$ \\
\hline Total cholesterol (mg/dL) & $997 \pm 48$ & $1,067 \pm 49$ \\
\hline HDL-cholesterol (mg/dL) & $30 \pm 2.7$ & $58 \pm 12^{\mathrm{a}}$ \\
\hline LDL-cholesterol (mg/dL) & $925 \pm 50$ & $914 \pm 53$ \\
\hline Triglycerides $(\mathrm{mg} / \mathrm{dL})$ & $216 \pm 45$ & $439 \pm 166$ \\
\hline Alkaline phosphatases (U/L) & $24 \pm 0.78$ & $26 \pm 3.1$ \\
\hline $\operatorname{ALT}(\mathrm{U} / \mathrm{L})$ & $72 \pm 9.9$ & $65 \pm 11$ \\
\hline $\operatorname{AST}(\mathrm{U} / \mathrm{L})$ & $36 \pm 8.1$ & $69 \pm 33$ \\
\hline
\end{tabular}

Data are means \pm SEM for $n$ animals. ${ }^{a} \mathrm{P}<0.05$ versus SFD (Mann-Whitney U test). Abbreviations: (ALT) alanine aminotransferase, (AST) aspartate aminotransferase and standard-fat diet (SFD).

\section{Liver function}

Plasma levels of the liver enzymes alkaline phosphatases, AST and ALT were not different after SFD or HFD feeding for 1 year (Table 2). The higher liver weight after HFD was associated with significantly higher hepatic triglyceride content (Figure 1A). However, only mild steatosis was apparent in liver sections after 1 year of HFD (Figure 1B). This is supported by similar hepatic gene expression of markers of steatosis (FAS, CD36) and or fibrosis (TIMP-1, TGF- $\beta$, Col1a1, $\alpha$-SMA) after exposure to both diets. Expression analysis of inflammatory markers revealed higher expression and lower $\Delta \mathrm{Ct}$ only for TNF- $\alpha$ on HFD (Table 3).

Table 3: Effect of a high-fat and high-cholesterol diet (HFD) on hepatic gene expression in coronary atherosclerosis- and myocardial infarction-prone Watanabe heritable hyperlipidemic (WHHLMI) rabbits

\begin{tabular}{|c|c|c|}
\hline & $\operatorname{SFD}(n=4-8)$ & $\operatorname{HFD}(\mathrm{n}=5-7)$ \\
\hline \multicolumn{3}{|c|}{ Steatosis } \\
\hline FAS & $7.9 \pm 0.31$ & $7.5 \pm 0.18$ \\
\hline CD36 & $1.5 \pm 0.11$ & $1.6 \pm 0.30$ \\
\hline \multicolumn{3}{|c|}{ Inflammation } \\
\hline TNF- $\alpha$ & $11 \pm 0.15$ & $9.7 \pm 0.57^{\mathrm{a}}$ \\
\hline IL-6 & $13 \pm 0.35$ & $12 \pm 1.0$ \\
\hline MCP-1 & $11 \pm 0.36$ & $10 \pm 0.42$ \\
\hline Arg-1 & $-0.76 \pm 0.47$ & $0.59 \pm 0.44$ \\
\hline \multicolumn{3}{|l|}{ Fibrosis } \\
\hline TIMP-1 & $7.6 \pm 0.31$ & $7.6 \pm 0.54$ \\
\hline TGF- $\beta$ & $6.3 \pm 0.14$ & $6.8 \pm 0.24$ \\
\hline Colla1 & $5.8 \pm 0.26$ & $6.5 \pm 0.34$ \\
\hline$\alpha$-SMA & $5.9 \pm 0.34$ & $6.3 \pm 0.34$ \\
\hline
\end{tabular}

Data are means $\pm \mathrm{SEM}$ of $\Delta \mathrm{Ct}\left(\mathrm{Ct}_{\text {target gene }}-\mathrm{Ct}_{\text {housekeeping gene }}\right)$ for $\mathrm{n}$ animals. ${ }^{a} \mathrm{P}<0.05$ versus standard-fat diet (SFD; Mann-Whitney $U$ test). Abbre- 

10- 17 .

viations: (FAS) fatty acid synthase, (CD36) cluster of differentiation 36 , (TNF- $\alpha$ ) tumour necrosis factor- $\alpha$, (IL-6) interleukin-6, (MCP-1) monocyte chemoattractant protein-1, (Arg-1) arginase-1, (TIMP-1) tissue inhibitor of metalloproteinases- 1 , (TGF- $\beta$ ) transforming growth factor- $\beta$, (Col1a1) $\alpha 1$ type 1 collagen, $(\alpha$-SMA) $\alpha$-smooth muscle actin. As housekeeping gene $\beta$-actin was used.
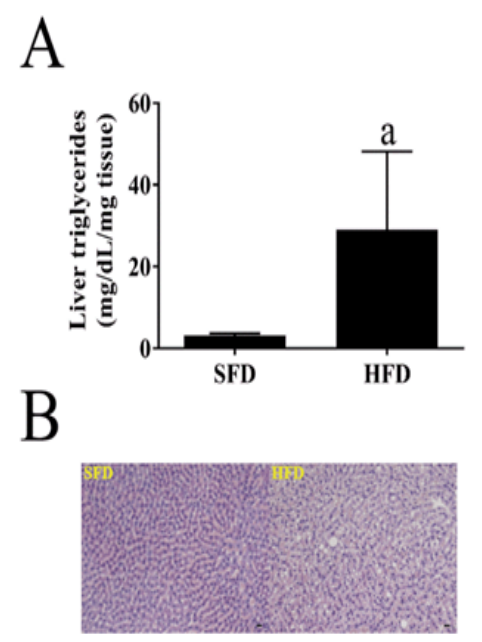

Figure 1: Effect of a HFD on hepatosteatosis in WHHLMI rabbits.

(A) Liver triglyceride content in coronary atherosclerosis- and myocardial infarction-prone Watanabe hereditary hyperlipidemic (WHHLMI) rabbits exposed for 12 months to either a standard-fat diet (SFD) or a high-fat diet enriched with cholesterol (HFD). Data are means \pm SEM of 7 (SFD) or 8 (HFD) animals. ${ }^{\mathrm{P}}<0.001$ versus SFD (Mann-Whitney U test). (B) H\&E (general morphology) staining of liver sections. Scale bar $=20 \mu \mathrm{m}$. Magnification: 200x.

\section{Aortic atherosclerosis}

The aortic surface in both SFD- and HFD-fed animals was covered with atherosclerotic plaques for more than $90 \%$ (ex vivo and histological sections, data not shown). The degree of stenosis in the thoracic aorta at the level of the diaphragm was not influenced by long-term HFD administration (SFD: $55 \pm 2.1 \%$ versus HFD: $55 \pm 1.8 \% ; \mathrm{P}=0.86 ; \mathrm{n}=3-4$ ) (Figure $2 \mathrm{~A}, \mathrm{D})$. Calcification of vascular atherosclerotic lesions as characterized by Von Kossa staining (Figure 2B, E) was not different $(\mathrm{P}=0.10 ; \mathrm{n}$ $=3$ ) between aorta sections of HFD-treated $(1.5 \pm 0.33 \%)$ versus SFD-exposed $(3.7 \pm 0.47 \%)$ WHHLMI rabbits. This coincided with an unchanged infiltration of macrophages (Figure 2C, F) in these sections (HFD: $13 \pm 6.5 \%$ versus $\mathrm{SFD}: 19 \pm 3.5 ; \mathrm{P}=0.63$; $\mathrm{n}=3-4)$.
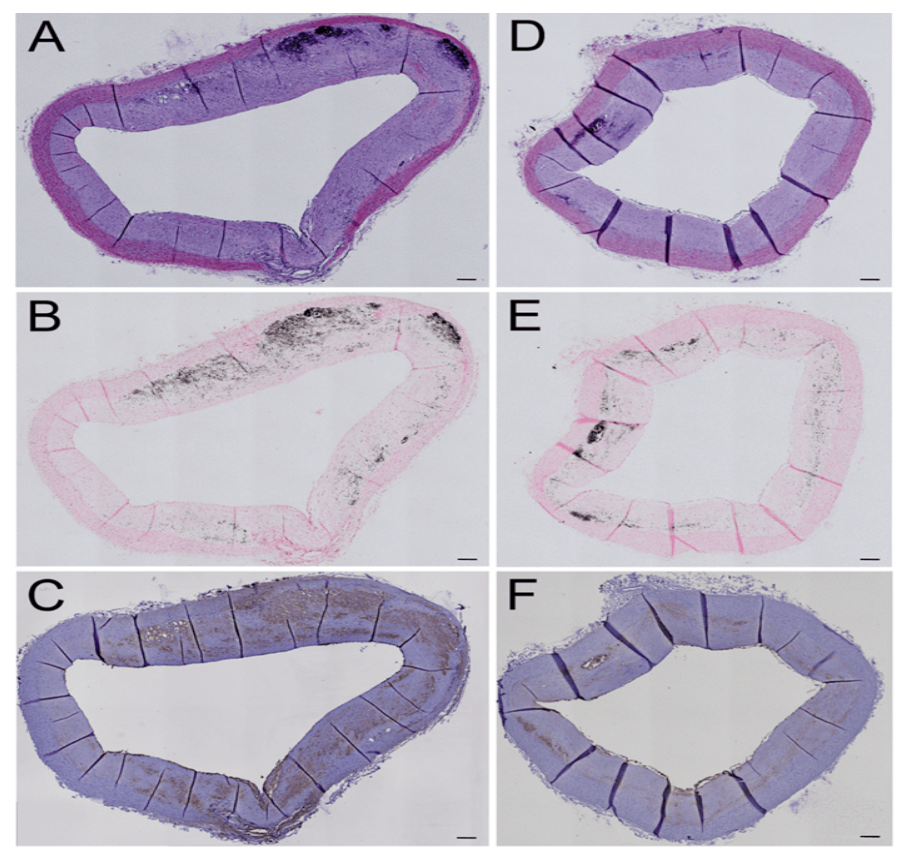

Figure 2: Effect of a HFD on atherogenesis in WHHLMI rabbits.

Aorta sections of coronary atherosclerosis- and myocardial infarction-prone Watanabe hereditary hyperlipidemic (WHHLMI) rabbits exposed for 12 months to either a standard-fat diet (SFD; A-C) or a high-fat diet enriched with cholesterol (HFD; D-F) were stained with hematoxylin and eosin (A, D) to study general morphology, with Von Kossa staining to identify plaque calcifications (black areas in B and E) and with RAM-11 antibodies to show macrophage infiltration (brown color in $\mathrm{C}$ and F) in the atherosclerotic plaques. Magnification: 100x. Scale bar $=200 \mu \mathrm{m}$.

\section{Cardiac function and myocardial fibrosis}

Cardiac function analysis of WHHLMI rabbits via MRI showed that HFD-fed rabbits had a significantly reduced SV and EF after one year of diet as compared to baseline, while SFD-exposed rabbits only displayed a slightly lower SV at the end versus the start of the study (Table 4). Representative mid-ventricle slices obtained by cMRI of both rabbit groups show a similar heart structure at baseline and at one year post-feeding for both end-diastolic and end-systolic phases (Figure 3). Echocardiographic analysis did not reveal differences in cardiac function parameters (Figure 4) between the two groups at baseline and after 1 year feeding, which was confirmed by representative mitral valve, mid-ventricle at papillary muscle level, apical and long axis slices after 12 months exposure to the diet (Figure 5).

SFD

HFD
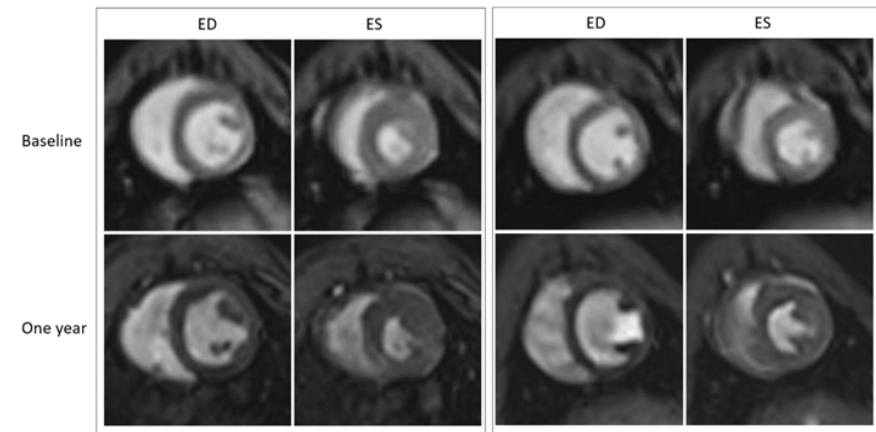

Figure 3: Longitudinal evaluation of cardiac function of WHHLMI rabbits by MRI

Effect of a 12 month exposure to a high-fat diet enriched with cholester- 
ol (HFD) on cardiac function in coronary atherosclerosis- and myocardial infarction-prone Watanabe heritable hyperlipidemic (WHHLMI) rabbits analyzed via magnetic resonance imaging (MRI). End-diastolic (ED) and end-systolic (ES) cine MRI scans were obtained at baseline and one year after exposure to a HFD or a standard-fat diet (SFD).
A

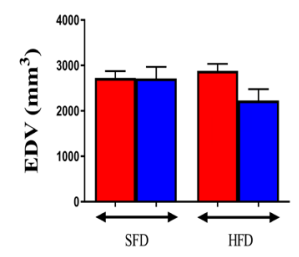

C

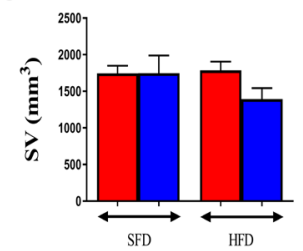

E

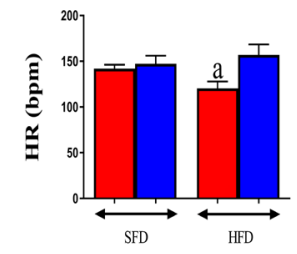

B
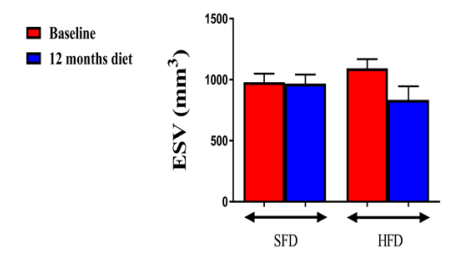

D
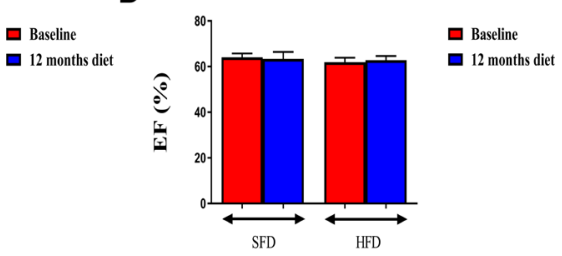

$\mathbf{F}$

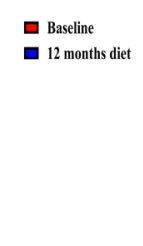

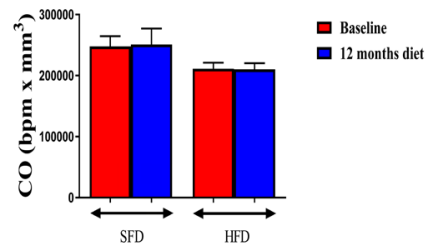

Figure 4: Effect of a HFD on cardiac function in WHHLMI rabbits by echocardiography

Effect of a 12 month exposure to high-fat diet enriched with cholesterol (HFD) on cardiac function in coronary atherosclerosis- and myocardial infarction-prone Watanabe hereditary hyperlipidemic rabbits. Control rabbits received a standard-fat diet (SFD). Cardiac function parameters that were evaluated via transthoracic echocardiography were: end-diastolic volume (EDV; A); end-systolic volume (ESV; B); stroke volume $(\mathrm{SV} ; \mathrm{C})$; ejection fraction (EF; D); heart rate (HR; E) and cardiac output $(\mathrm{CO} ; \mathrm{F})$. Data are means \pm SEM of 6 rabbits. ${ }^{\mathrm{a}}<0.05$ versus $\mathrm{SFD}$.

Table 4: Effect of a high-fat and high-cholesterol diet (HFD) on cardiac function as assessed via magnetic resonance imaging in coronary atherosclerosis- and myocardial infarction-prone Watanabe heritable hyperlipidemic (WHHLMI) rabbits

\begin{tabular}{|l|l|l|l|l|}
\hline \multirow{2}{*}{} & \multicolumn{2}{|c|}{ SFD } & \multicolumn{2}{c|}{ HFD } \\
\cline { 2 - 5 } & Baseline & $\begin{array}{c}12 \text { months } \\
\text { diet }\end{array}$ & \multicolumn{1}{|c|}{ Baseline } & $\begin{array}{c}12 \text { months } \\
\text { diet }\end{array}$ \\
\hline EDV $(\mathrm{ml})$ & $3.3 \pm 0.18$ & $3.0 \pm 0.13$ & $3.1 \pm 0.17$ & $2.9 \pm 0.13$ \\
\hline ESV $(\mathrm{ml})$ & $1.3 \pm 0.081$ & $1.3 \pm 0.076$ & $1.2 \pm 0.099$ & $1.3 \pm 0.067$ \\
\hline SV $(\mathrm{ml})$ & $2.0 \pm 0.11$ & $1.1 \pm 0.080^{\mathrm{b}}$ & $1.9 \pm 0.089$ & $\begin{array}{l}1.6 \pm \\
0.072^{\mathrm{bb}}\end{array}$ \\
\hline EF $(\%)$ & $61 \pm 0.94$ & $57 \pm 1.3$ & $61 \pm 1.6$ & $55 \pm 0.85^{\text {bb }}$ \\
\hline HR $(\mathrm{bpm})$ & $146 \pm 8.4$ & $136 \pm 7.1$ & $137 \pm 7.0$ & $133 \pm 7.1$ \\
\hline $\begin{array}{l}\mathrm{CO}(\mu \mathrm{l} x \\
\text { bpm })\end{array}$ & $\begin{array}{l}0.30 \pm \\
0.020\end{array}$ & $\begin{array}{l}0.23 \pm \\
0.017\end{array}$ & $0.26 \pm$ & $0.21 \pm$ \\
\hline
\end{tabular}

Data are means \pm SEM of 8 animals/group. ${ }^{\mathrm{b}} \mathrm{P}<0.05$ and ${ }^{\mathrm{bb}} \mathrm{P}<0.01$ versus baseline (Mann-Whitney U test). Abbreviations: (SFD) standard-fat diet, (EDV) end-diastolic left ventricular (LV) volume, (ESV) end-systolic LV volume, (SV) stroke volume, (EF) ejection fraction, (HR) heart rate), (CO) cardiac output.
SFD
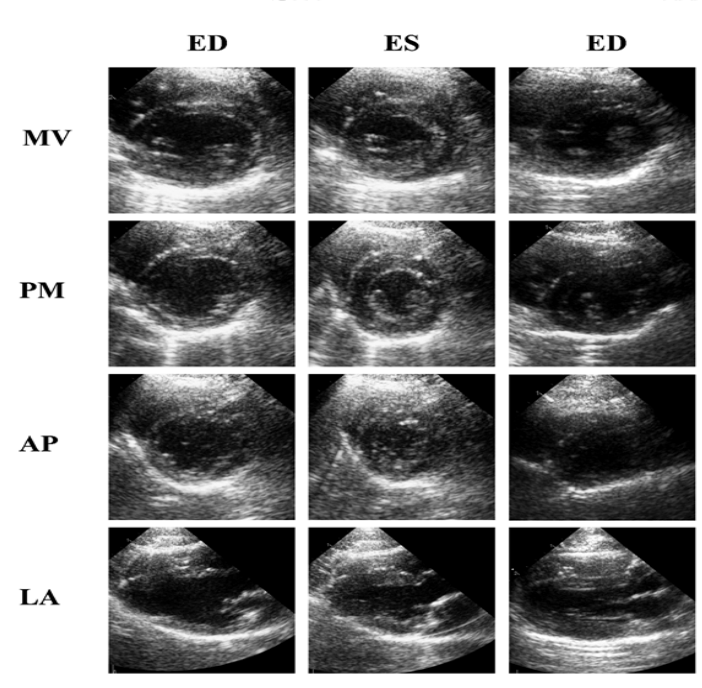

ES

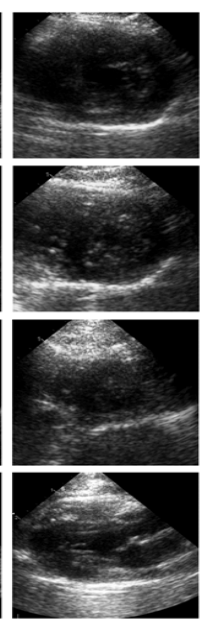

Figure 5: Longitudinal evaluation of cardiac function of WHHLMI rabbits by echocardiography

Effect of a 12 month exposure to a high-fat diet enriched with cholesterol (HFD) on cardiac function in coronary atherosclerosis- and myocardial infarction-prone Watanabe heriditary hyperlipidemic (WHHLMI) rabbits analyzed using transthoracic echocardiography. End-diastolic (ED) and end-systolic (ES) echocardiography images at the short axis (mitral valve (MV), papillary muscle (PM) and apex (AP)) and long axis (LA) level of WHHLMI rabbits exposed 12 months to a HFD or a standard-fat diet (SFD) as control.

Only one rabbit on SFD showed extensive fibrosis of the LV myocardium after 1 year feeding (Figure 6), which may represent the morphological change in chronic ischemic heart disease. Cardiac troponin $\mathrm{T}$ levels for all animals were below the detection limit of the assay at both time points (data not shown).
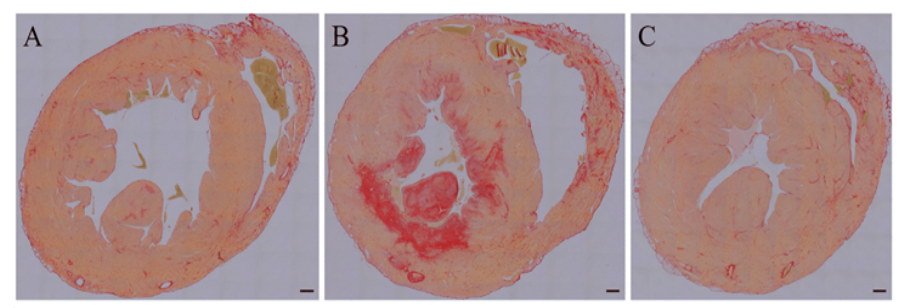

Figure 6: Myocardial fibrosis in WHHLMI rabbits

Mid-ventricular sections of coronary atherosclerosis- and myocardial infarction-prone Watanabe hereditary hyperlipidemic (WHHLMI) rabbits exposed for 12 months to either a standard-fat diet (SFD; A-B) or a high-fat diet enriched with cholesterol (HFD; C) were stained with Sirius Red to show myocardial fibrosis. The mid-ventricular section of one rabbit on SFD (B) showed extensive fibrosis of the septal, anterior, and lateral LV myocardium and of the anterior and posterior papillary muscle. Magnification: $5 x$. Scale bar $=1 \mathrm{~mm}$.

\section{Discussion}

Myocardial infarction-prone Watanabe heritable hyperlipidemic (WHHLMI) rabbits spontaneously develop severe coronary atherosclerosis and MI and are therefore used to investigate the pathogenic mechanisms leading to atherosclerotic plaque development ${ }^{[12]}$ and to identify potential new therapeutic interventions $^{[13]}$. High plasma LDL levels compatible with a defect in LDL receptor function characterize these animals. The present 
study shows, independent of diet feeding, considerably elevated plasma LDL concentrations in both rabbit groups. WHHLMI rabbits show clinical features of the metabolic syndrome with age: middle-aged rabbits showed more visceral fat accumulation and a higher accumulation of RAM-11 positive macrophages in the MES AT depot and lower plasma insulin levels as compared to adult WHHLMI rabbits ${ }^{[4]}$.

Obesity and insulin resistance can be induced in experimental animals by exposing them to particular high-fat diets. The diet that was used in this study to induce obesity and insulin resistance was applied in two earlier studies. Aragno et al. demonstrated that administration of the HFD to New Zealand White (NZW) rabbits for 12 weeks increased body weight (11\%), fasting glucose (1.5-fold) and insulin (5.9-fold) levels, and the homeostatic model assessment of insulin resistance in$\operatorname{dex}(9.6 \text {-fold })^{[6]}$. Furthermore, diet administration reduced basal contractility of the papillary muscles in the NZW rabbits ${ }^{[6]}$. In another study by Carroll et al. body weight was $44 \%$ higher after 12 weeks exposure of NZW rabbits to a HFD versus a SFD ${ }^{[7]}$. Obesity resulted in markedly increased serum creatinine kinase MB levels, a biomarker of cardiac tissue injury ${ }^{[7]}$. Exposing WHHL rabbits for 16 weeks to a HFFD versus a control diet however, did not affect body weight, but did lead to more SC and visceral fat accumulation, higher plasma and hepatic triglyceride levels, impaired glucose tolerance and accelerated aortic and coronary atherosclerosis ${ }^{[5]}$. In this study, we exposed WHHLMI rabbits for one year to this HFD to slowly induce obesity, and to investigate whether these pathological conditions would enhance atherogenesis and affect cardiac function, leading to an accelerated mortality. As reported, the administration of the HFD versus the SFD did indeed induce obesity as we noticed a $37 \%$ increase in body weight, more fat accumulation (280\% for SC and 521\% for GON fat depot, respectively) and more hepatosteatosis. Although fasting blood glucose and plasma insulin levels after 12 months feeding did not differ between the two rabbit groups, the HFD-fed animals were less glucose tolerant in comparison to SFD-fed rabbits during an iv glucose tolerance test. Despite the induction of obesity, especially visceral adiposity (more mesenteric fat accumulation was visually observed as well; data not shown), and glucose intolerance, the HFD did not affect the rate of atherogenesis and its associated induction of MI. Indeed, SFD-fed and HFD-fed animals did not show differences in overall survival time and thoracic aorta lumen stenosis. The surfaces of the entire aorta tract in both animal groups were more than $90 \%$ covered with atherosclerotic plaques (data not shown), which is known for WHHLMI rabbits older than 10 months ${ }^{[14]}$. In both groups, the surface of the thoracic and aortic arch (data not shown) aorta sections were almost completely covered by plaques. Cardiac function was only slightly affected by the HFD after 12 months feeding as characterized by a slight significant decrease in EF. However, only one of the surviving animals of the SFD-fed group displayed extensive fibrosis of the septal, anterior, and lateral LV myocardium and of the anterior and posterior papillary muscle, suggestive of the morphological change in chronic ischemic heart disease.

The reason why the HFD did not accelerate atherogenesis and subsequent development of MI might be multifactorial. To stimulate atherogenesis, experimental diets enriched in cholesterol are used ${ }^{[15-17]}$. Our 57T4 diet was enriched with 32 ppm (or $0.003 \%$ ) cholesterol, while the SFD (5322 diet) was devoid of cholesterol. Most atherogenic diets are enriched with 0.015 $\%$ up to $2 \%$ cholesterol $^{[15-17]}$ and therefore the lower amount of cholesterol in our diet may partially explain the lack of atheroma progression. Exposing rabbits for one year to a HFD would expected to be long enough to aggravate atherogenesis in a model of familial hypercholesterolemia. Indeed, exposure of WHHL rabbits for 16 weeks to a HFFD accelerated aortic as wells as coronary atherosclerosis ${ }^{[5]}$. However, they did find that glucose intolerance did not get worse over time and that the level of atherosclerotic lesions covering the aortic surface saturated at 16 weeks of HFFD feeding ${ }^{[5]}$. This study would suggest that with age hypercholesterolemia in WHHL animals would mask the atherogenic effect of the HFFD diet. In our study, we did observe that the surface of the entire tract was almost completely covered in both diet regimens. However, glucose intolerance was only observed after 12 months and not after 41 weeks of HFD feeding. Early stages of HFD feeding (11 weeks, 3 and 6 months) were accompanied with lower fasting blood glucose levels, while in the study of Ning et al..$^{[5]}$ blood glucose levels were not affected by the HFFD. Plasma lipid levels at these earlier time points were however not determined, which is a limitation in our study. Another limitation is that the extent of coronary atherosclerosis was not investigated. As the HFD did not accelerate atherogenesis and development of MI after 12 months of feeding (age of animals: \pm 18 -19 months), we believe it is plausible that coronary atherosclerosis was not advanced enough in both groups to induce MI. Indeed, the reported cumulative incidence of fatal MI in male WHHLMI rabbits is roughly $20 \%$ between the age of 11 and 20 months $^{[14]}$. In our study $16 \%$ (3/19) animals died before the end of the study, which is in agreement with this.

\section{Conclusion}

On SFD, WHHLMI rabbits present with 55\% thoracic aorta lumen stenosis, but they appear resistant to HFD-induced diabetes or accelerated atherosclerosis.

Acknowledgements: We thank C. Vranckx, I. Vorsters and L. Hendrickx for expert technical assistance. We are grateful to Prof. Dr. Shiomi (Institute for Experimental Animals, Kobe University School of Medicine, Kobe, Japan) for providing the WHHLMI rabbits.

Financials: The Center for Molecular and Vascular Biology, and Radiology were supported by an IWT O\&O grant (ICD 668894).

Conflict of Interests: All authors declare no conflict of interests.

\section{References}

1. Gleissner, C.A. Translational atherosclerosis research: From experimental models to coronary artery disease in humans. (2016) Atherosclerosis 248: 110-116. Pubmed | Crossref | Others

2. Baumgartner, C., Brandl, J., Munch, G., et al. Rabbit models to study atherosclerosis and its complications - Transgenic 
vascular protein expression in vivo. (2016) Prog Biophys Mol Biol 121(2): 131-141.

Pubmed | Crossref | Others

3. Shiomi, M., Ito T., Yamada S., et al. Development of an animal model for spontaneous myocardial infarction (WHHLMI rabbit). (2003) Arterioscler Thromb Vasc Biol 23(7): 1239-1244.

\section{Pubmed | Crossref | Others}

4. Shiomi, M., Kobayashi T., Kuniyoshi N., et al. Myocardial infarction-prone Watanabe heritable hyperlipidemic rabbits with mesenteric fat accumulation are a novel animal model for metabolic syndrome. (2012) Pathobiology 79(6): 329338.

\section{Pubmed | Crossref | Others}

5. Ning, B., Wang X., Yu Y., et al. High-fructose and highfat diet-induced insulin resistance enhances atherosclerosis in Watanabe heritable hyperlipidemic rabbits. (2015) Nutr Metab (Lond) 12: 30.

Pubmed | Crossref | Others

6. Aragno, M., Meineri, G., Vercellinatto, I., et al. Cardiac impairment in rabbits fed a high-fat diet is counteracted by dehydroepiandrosterone supplementation. (2009) Life Sci 85(1-2): 77-84.

Pubmed | Crossref | Others

7. Carroll, J.F., Tyagi, S.C. Extracellular matrix remodeling in the heart of the homocysteinemic obese rabbit. (2005) Am J Hypertens 18(5 Pt 1): 692-698.

Pubmed | Crossref | Others

8. Feng, Y., Chen, F., Yin, T., et al. Pharmacologic Effects of Cannabidiol on Acute Reperfused Myocardial Infarction in Rabbits: Evaluated With 3.0T Cardiac Magnetic Resonance Imaging and Histopathology. (2015) J Cardiovasc Pharmacol 66(4): 354-363.

Pubmed | Crossref | Others

9. Stypmann, J., Engelen, M.A., Troatz, C., et al. Echocardiographic assessment of global left ventricular function in mice. (2009) Lab Anim 43(2): 127-137.

Pubmed | Crossref | Others

10. Wong, T., Hildebrandt, M.A., Thrasher, S.M., et al. Divergent metabolic adaptations to intestinal parasitic nematode infection in mice susceptible or resistant to obesity. (2007) Gastroenterology 133(6): 1979-1988.

Pubmed | Crossref | Others

11. Hemmeryckx, B., Van Hove, C.E., Fransen, P., et al. Progression of the prothrombotic state in aging Bmal1-deficient mice. (2011) Arterioscler Thromb Vasc Biol 31(11): 2552-2559.

Pubmed | Crossref | Others
12. Nagasaka, R., Koike, T., Tsukada, N., et al. The Coronary Artery Running Pattern is One of the Causes of Individual Differences in the Progression of Coronary Atherosclerosis in WHHLMI Rabbits, an Animal Model for Coronary Atherosclerosis. (2017) J Atheroscler Thromb 25(5): 393-404. Pubmed | Crossref | Others

13. Tamura, S., Koike, Y., Takeda, H., et al. Ameliorating effects of D-47, a newly developed compound, on lipid metabolism in an animal model of familial hypercholesterolemia (WHHLMI rabbits). (2018) Eur J Pharmacol 822:147-153.

Pubmed | Crossref | Others

14. Ito, T., Yamada, S., Shiomi, M. Progression of coronary atherosclerosis relates to the onset of myocardial infarction in an animal model of spontaneous myocardial infarction (WHHLMI rabbits). (2004) Exp Anim 53(4): 339-346.

Pubmed | Crossref | Others

15. Amani, M., Darbin, A., Pezeshkian, M., et al. The role of cholesterol-enriched diet and paraoxonase 1 inhibition in atherosclerosis progression. (2017) J CardiovascThorac Res 9(3): 133-139.

Pubmed | Crossref | Others

16. Hu, H., Gao, Y., Tang, J., et al. Effect of a high-cholesterol diet on lipoprotein metabolism and xanthoma formation in rabbits. (2017) J Cosmet Dermatol.

Pubmed | Crossref | Others

17. Ibrahim, M., Ahmed, I.A., Mikail, M.A., et al. Baccaureaangulata fruit juice reduces atherosclerotic lesions in diet-induced Hypercholesterolemic rabbits. (2017) Lipids Health Dis 16(1): 134. Pubmed | Crossref | Others

Submit your manuscript to Ommega Publishers and we will help you at every step:

- We accept pre-submission inquiries

- Our selector tool helps you to find the most relevant journal

- We provide round the clock customer support

- Convenient online submission

- Thorough peer review

- Inclusion in all major indexing services

- Maximum visibility for your research

Submit your manuscript at OMMEGA Publishers https://www.ommegaonline.org/submit-manuscript 